Supporting Information

\title{
Volatile Organic Compound Sensing Properties of Parylene E: Thermal Transition and Sorption Kinetics
}

\author{
Kenichi Morimoto, ${ }^{*}$ Cheng-Han Yeh, Takahiro Mito, Yuji Suzuki \\ Department of Mechanical Engineering, The University of Tokyo, 7-3-1, Hongo, Bunkyo-ku, Tokyo 113- \\ 8656, Japan \\ *Corresponding author: morimoto@mesl.t.u-tokyo.ac.jp
}




\section{S.1 DSC thermograms of parylene C}
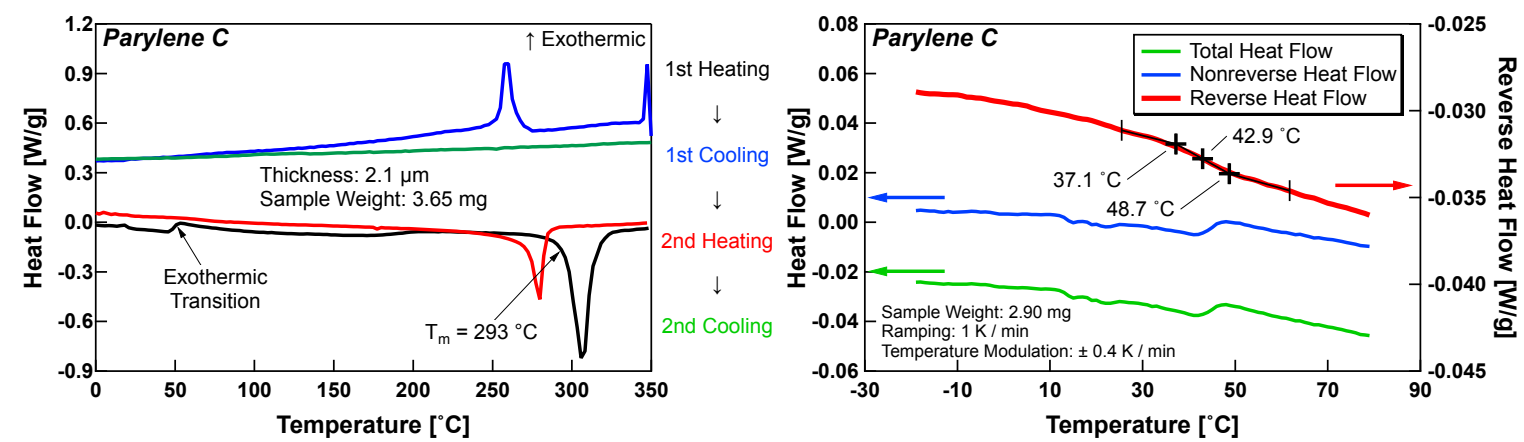

Figure S1. Thermograms for parylene C from standard DSC (left) and TMDSC (right) measurements. The test condition is similar to Figure 1 for parylene E. In DSC measurement (left), an exothermic step of heat flow is found from 46 to $54{ }^{\circ} \mathrm{C}$ in the first heating cycle. The step disappears from the second heating cycle. The melting transition of parylene $\mathrm{C}$ occurs at $293{ }^{\circ} \mathrm{C}$, which agrees with literature values ${ }^{[5,25-27]}$. In TMDSC measurement (right), an endothermic step occurs in the range of $37.1{ }^{\circ} \mathrm{C}$ to $48.7{ }^{\circ} \mathrm{C}$, and thus the glass transition of parylene $\mathrm{C}$ is identified. The glass transition temperature of parylene $\mathrm{C}$ is estimated to be $42.9^{\circ} \mathrm{C}$. It is clearly seen that an exothermic step appears in the total heat flow and the non-reverse heat flow, which is also observed in the standard DSC. The exothermic peak of parylene $\mathrm{C}$ at around $50{ }^{\circ} \mathrm{C}$ is judged to represent the enthalpy relaxation due to the cross-linking at low temperature.

\section{S.2 Viscoelasticity measurement by QCM-D}
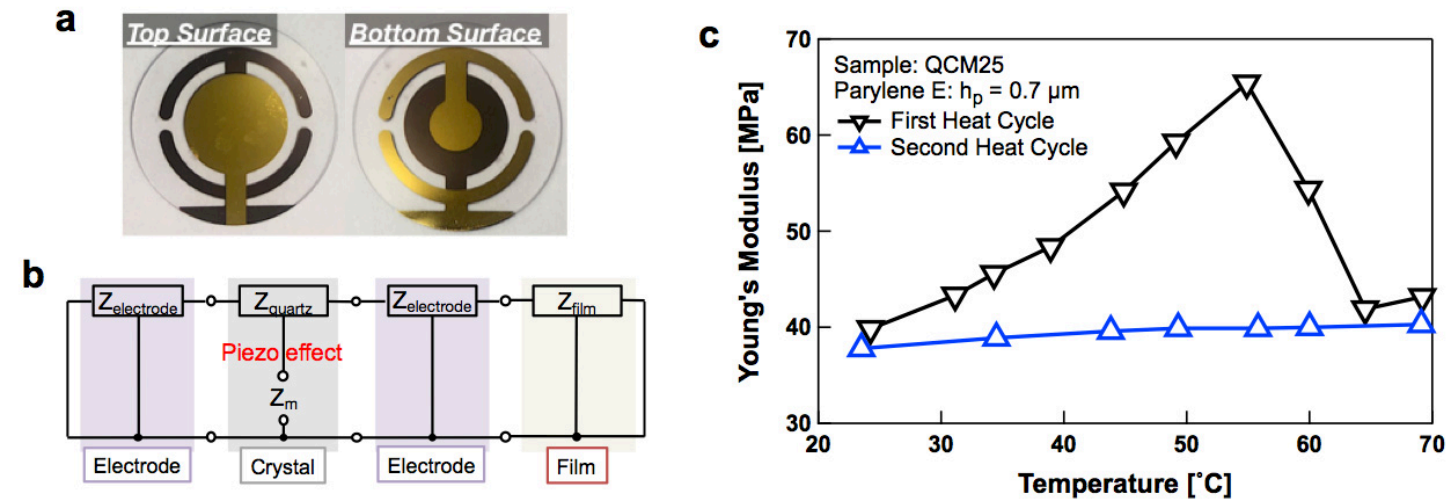

Figure S2. Measurement of viscoelastic properties of parylene E. a) photos of the quartz crystal deposited with parylene E. b) Mason equivalent circuit. c) Young's modulus versus temperature. The peak of the loss ratio locates at around $55^{\circ} \mathrm{C}$, where Young's modulus has its peak in the first heat cycle. There is no significant change during the second heat cycle, and it is considered that the irreversibility in the present measurement is in accordance with the thermal transition behavior in DSC measurements.

Viscoelastic properties of the thin parylene E film are evaluated by quartz crystal microbalance with dissipation (QCM-D) before and after parylene E deposition. The relationship between the frequency shift $\Delta \mathrm{f}$ and the mass loading per unit area $\Delta \mathrm{m}$ is expressed by Sauerbrey equation: ${ }^{[\mathrm{S} 1]}$ 


$$
\Delta f=-\frac{2 \mathrm{~N} f_{\mathrm{n}=1}^{2}}{\sqrt{\mu_{\mathrm{q}} \rho_{\mathrm{q}}}} \Delta m=\frac{2 \mathrm{n} f_{\mathrm{n}=1}^{2}}{\sqrt{Z_{\mathrm{q}}}} \Delta m=-C_{\mathrm{f}} \cdot \Delta m,
$$

where $\mu_{\mathrm{q}}, \rho_{\mathrm{q}}, Z_{\mathrm{q}}$ are the viscosity, density and the impedance of the quartz, respectively, and $C_{\mathrm{f}}$ is the prefactor combining the properties of the quartz. In the present measurement, an AT-cut quartz with 17.7 $\mathrm{ng} / \mathrm{cm}^{2}$ of the mass change by $1 \mathrm{~Hz}$ of the frequency shift ${ }^{[\mathrm{S} 2]}$ was used. The admittance of the quartz around the resonant frequency can be expressed by Butterworth-van Dyke (BVD) equivalent circuit ${ }^{[30]}$ as:

$$
\begin{gathered}
Y=\frac{1}{Z}=G+i B \\
G=\frac{R_{\mathrm{m}}}{R_{\mathrm{m}}^{2}+\left(\omega L_{\mathrm{m}}-1 / \omega C_{\mathrm{m}}\right)^{2}}, \\
B=\omega C_{0}-\frac{\omega_{m}-1 / \omega C_{\mathrm{m}}}{R_{\mathrm{m}}^{2}+\left(\omega L_{\mathrm{m}}-1 / \omega C_{\mathrm{m}}\right)^{2}},
\end{gathered}
$$

where $G$ is the conductance, $B$ is the susceptance, $R_{\mathrm{m}}$ is the damping energy loss per oscillation, $L_{\mathrm{m}}$ is the inertia of the quartz, $C_{\mathrm{m}}$ is the energy stored per oscillation, and $C_{0}$ is the capacitance of the dielectric crystal sandwiched between the electrodes. For a quartz with a resonant frequency of $5 \mathrm{MHz}$, the parameters in BVD circuit are estimated to be $R_{\mathrm{m}}=20 \Omega, L_{\mathrm{m}}=30 \mathrm{mH}, C_{\mathrm{m}}=30 \mathrm{fF}$, and $C_{0}=$ $20 \mathrm{pF}^{[\mathrm{S} 2]}$.

The Mason equivalent circuit as shown in Figure S2b is applied to separate the film properties from an electrode-deposited quartz with the film loaded. The mechanical impedance $\mathrm{Z}_{\mathrm{m}}$ is given by: ${ }^{[31]}$

$$
Z_{\mathrm{m}}=-\frac{i Z_{\mathrm{q}}}{2} \cot \left(\frac{k_{\mathrm{q}} d_{\mathrm{q}}}{2}\right)+\frac{Z_{\text {front }}}{2}
$$

where $k_{\mathrm{q}}=\omega / v_{\mathrm{q}}$ is the wave number of the transverse sound with the sound speed in the quartz $v_{\mathrm{q}}=3325 \mathrm{~m} / \mathrm{s}, d_{\mathrm{q}}$ is the thickness of the quartz, and $Z_{\text {front }}$ is the impedance of the front side of the quartz. Since the impedance of front side electrodes is much smaller than $Z_{\mathrm{q}}$, the resonance conditions are close to the poles of the tangent, and $Z_{\mathrm{m}}$ is equal to 0 at the resonant frequency. Then the term inside cotangent is

$$
\frac{k_{\mathrm{q}} d_{\mathrm{q}}}{2}=\frac{\mathrm{n} \pi}{2}(1+\varepsilon), \quad \mathrm{n}=1,3,5, \ldots
$$

where $\varepsilon=\varepsilon^{\prime}+\mathrm{i} \varepsilon^{\prime \prime}$ is a small complex number and $\mathrm{n}$ is the overtone number. Thus,

$$
\mathrm{i} \tan \left(\frac{\mathrm{n} \pi}{2} \varepsilon\right)=-\frac{1}{Z_{\mathrm{q}}} Z_{\text {front }}
$$

After the film deposition, Eq. (S7) becomes: 


$$
\tan \left(\frac{\mathrm{n} \pi}{2} \varepsilon\right)=-\frac{1}{Z_{\mathrm{q}}} \frac{Z_{\mathrm{f}} \tan \left(k_{\mathrm{f}} d_{\mathrm{f}}\right)+Z_{\mathrm{e}} \tan \left(k_{\mathrm{e}} d_{\mathrm{e}}\right)}{1-Z_{\mathrm{f}} / Z_{\mathrm{e}} \tan \left(k_{\mathrm{f}} d_{\mathrm{f}}\right) \tan \left(k_{\mathrm{e}} d_{\mathrm{e}}\right)}
$$

where the subscript $f$ denotes the parameters of the film and the subscript e denotes the parameters of the electrode. The temperature range of interest is from room temperature to $80{ }^{\circ} \mathrm{C}$, so that the electrodes are stiff and $1 / Z_{\mathrm{e}}$ is close to 0 . Also, the electrode thickness is in $\mu \mathrm{m}$-order so that $k_{\mathrm{e}} d_{\mathrm{e}} \ll 1$ and $\tan \left(k_{\mathrm{e}} d_{\mathrm{e}}\right)=0$. By defining $\varepsilon=\Delta f / f+\mathrm{i} \Delta \Gamma / f$, Eq. (S8) becomes

$$
\tan \left[2 \pi \cdot f \cdot d_{\mathrm{f}} \sqrt{\frac{\rho_{\mathrm{f}}}{G_{\mathrm{f}}}}\right]=-\frac{\mathrm{n} \pi Z_{\mathrm{q}}}{2 \sqrt{\rho_{\mathrm{f}} G_{\mathrm{f}}}} \frac{\Delta f+\mathrm{i} \Delta \Gamma}{f},
$$

where $G_{\mathrm{f}}=G^{\prime}+\mathrm{i} G^{\prime \prime}$ is the complex shear modulus of the thin film, and $G^{\prime}$ and $G^{\prime \prime}$ are the storage and the loss modulus, respectively.

The shear modulus can be calculated using the Maxwell model:

$$
\begin{gathered}
\frac{\partial \sigma^{*}(t)}{\partial t}+\frac{G}{\eta} \sigma^{*}(t)=G \frac{\partial \gamma^{*}(t)}{\partial t} \\
\sigma^{*}(t)=\left(G^{\prime}+G^{\prime \prime}\right) \gamma^{*}(t)
\end{gathered}
$$

The shear modulus and Young's modulus are then obtained as:

$$
\begin{gathered}
G=\frac{\left(G^{\prime}\right)^{2}+\left(G^{\prime \prime}\right)^{2}}{G^{\prime}}, \\
E=2(1+v) G,
\end{gathered}
$$

where the Poisson ratio is assumed to $v=0.4$.

\section{S3. Derivation of partition coefficient, volume fraction, and interaction parameter}

The capacitance change with VOC sorption is estimated by taking into account the thickness change due to the polymer swelling and the permittivity change that can be estimated from an analytical model proposed by Igreja \& Dias ${ }^{[\mathrm{S} 2]}$. The thickness change is assumed to be proportional to the volume fraction of VOC inside the polymer layer as:

$$
h_{\mathrm{p}}^{\prime}=h_{\mathrm{p}}\left(1+\phi_{1}\right)
$$

where $h_{\mathrm{p}}^{\prime}$ is the polymer thickness after VOC sorption, $h_{\mathrm{p}}$ is the initial thickness without VOC, and $\phi_{1}$ is the volume fraction of VOC inside the polymer layer.

When the volume fraction is low and the dispersion is uniform, the permittivity change of heterogeneous VOC-polymer system can be assumed to follow a relation similar to the Maxwell-Garnett mixing formula:

$$
\varepsilon_{\mathrm{m}}=\varepsilon_{\mathrm{VOC}}+\frac{3 \varepsilon_{\mathrm{VOC}}\left(\varepsilon_{\mathrm{p}}-\varepsilon_{\mathrm{VOC}}\right) \phi_{1}}{2 \varepsilon_{\mathrm{VOC}}+\varepsilon_{\mathrm{p}}-\phi_{1}\left(\varepsilon_{\mathrm{p}}-\varepsilon_{\mathrm{VOC}}\right)},
$$


where $\varepsilon_{\mathrm{VOC}}, \varepsilon_{\mathrm{p}}$, and $\varepsilon_{\mathrm{m}}$ denote the permittivity of the VOC, polymer, and the binary mixture, respectively. The volume fraction of VOC inside the polymer is given by

$$
\phi_{1}=\frac{V_{1}^{(\mathrm{p})}}{V_{2}}=\frac{M_{1} n_{1}^{(\mathrm{p})}}{\rho_{1, \ell} V_{2}},
$$

where $V_{1}^{(\mathrm{p})}$ is the volume of VOC inside the polymer, $V_{2}$ is the polymer volume, $M_{1}$ is the VOC molar mass, $n_{1}^{(\mathrm{p})}$ is the number of moles of VOC in the polymer, and $\rho_{1, \ell}$ is the VOC density in liquid phase ${ }^{[\mathrm{S} 3]}$. The volume fraction of VOC is related to the equilibrium ratio of the VOC concentration between the mobile and polymer phase. The ratio is termed as the partition coefficient $K$, which is given by:

$$
K=\frac{c_{1}^{(\mathrm{p})}}{c_{1}^{\text {(gas) }}}=\frac{n_{1}^{(\mathrm{p})} / V_{2}}{n_{1}^{\text {(gas) }} / V_{\mathrm{g}}}
$$

where $c_{1}^{(\mathrm{p})}$ is the VOC concentration inside the polymer, $c_{1}^{(\text {gas })}$ is the VOC concentration at mobile phase, $V_{2}$ is the polymer volume, $V_{\mathrm{g}}$ is the volume of the detector channel, and $n_{1}^{\text {(gas) }}$ is the number of moles of the carrier gas, respectively. From Eqs. (S16) and (S17), the volume fraction $\phi_{1}$ is related to the partition coefficient $K$ as:

$$
\phi_{1}=\frac{V_{1}^{(\mathrm{p})}}{V_{2}}=K \frac{M_{1} n_{1}^{(\mathrm{gas})}}{\rho_{1, \ell} V_{\mathrm{g}}}=K \frac{M_{1}}{\rho_{1, \ell}} \frac{c_{1}^{\text {(gas })} P_{\mathrm{g}}}{\mathrm{R} T},
$$

where $P_{\mathrm{g}}$ is the gas pressure in the mobile phase, $T$ is the temperature, and $\mathrm{R}$ is the gas constant.

The Flory-Huggins interaction parameter is introduced to evaluate the solvent-polymer interaction using the volume fraction. The interaction parameter can be expressed as a function of the volume fraction as:

$$
\chi_{12}=\left(1-\phi_{1}\right)^{-2}\left\{\ln \left[\frac{p_{1}}{p_{\text {sat }}}\right]-\ln \left[\phi_{1}\right]-\left(1-\phi_{1}\right)\right\},
$$

where $p_{1}$ and $p_{\text {sat }}$ are the partial vapor pressure and the saturated vapor pressure of the solvent at the given temperature, respectively. For small volume fraction, Eq. (S19) can be simplified to:

$$
\phi_{1}=\frac{p_{1}}{p_{\text {sat }}} \exp \left[-\left(\chi_{12}+1\right)\right] \text {. }
$$

Thus, the partition coefficient in Eq. (S18) can then be related to the volume fraction.

The partition coefficient can also be derived from QCM measurement. The relationship between the mass loading and frequency change is expressed by the Sauerbrey equation: ${ }^{[\mathrm{S} 1]}$

$$
\Delta f[\mathrm{~Hz}]=-C_{\mathrm{f}}\left[\frac{\mathrm{Hz} \cdot \mathrm{cm}^{2}}{\mu \mathrm{g}}\right] \cdot \frac{\Delta m}{A}\left[\frac{\mu \mathrm{g}}{\mathrm{cm}^{2}}\right],
$$

where $\Delta f$ is the change of the resonant frequency, $\Delta m$ is the mass loading per unit area, and $C_{\mathrm{f}}=-56.6$ ${ }^{[\mathrm{S} 2]}$ is the sensitivity factor for the quartz resonant at $5 \mathrm{MHz}$. The volume fraction can be derived as:

$$
\phi_{1}=\frac{\Delta m / A}{\rho_{1, \ell} \cdot h_{\mathrm{p}}}
$$

where $h_{\mathrm{p}}$ is the polymer thickness. The partition coefficient can then be estimated as well. 


\section{S4. Details of the capacitance measurement}
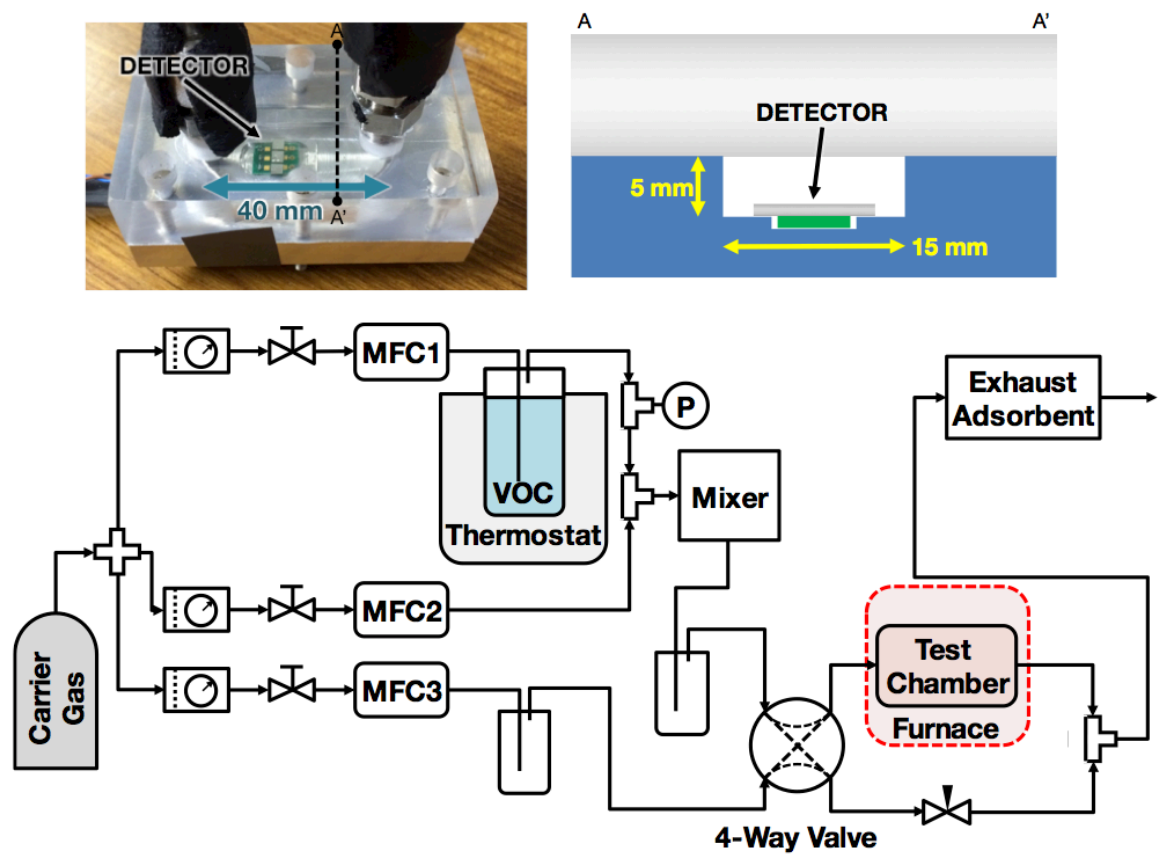

Figure S3. Experimental setup for measuring dynamic and equilibrium responses of the detector against VOC with controlled concentration. The detector is placed in the middle of the flow channel in the test chamber. The length of the flow channel is $40 \mathrm{~mm}$ with $75 \mathrm{~mm}^{2}$ of the cross section. Pure nitrogen is used as the carrier gas. Two inlets and two outlets from four independent flow paths are connected to a four-way valve, for switching the nitrogen flow and the nitrogen-VOC mixture flow to the detector. The flow rates are regulated by the mass flow controllers (MFC, Model 3200, Model 3660, Kofloc, Japan). The liquid VOC under the room temperature is prepared in the bubbler and diluted with the nitrogen to provide VOC samples with controlled concentration (ranging from 10 to $4000 \mathrm{ppm}$ ). The temperature of the bubbler is controlled by a water thermostat (LTB-125, As One, Japan), while the test chamber is controlled by a convection oven (DXS200, Yamato, Japan).

Table S1. Permittivity $(\varepsilon) \&$ solubility parameter $(\delta)$ of toluene, PDMS, and parylene E.

\begin{tabular}{cccc}
\hline Material & $\varepsilon$ & $\delta\left[\left(\mathrm{cal} / \mathrm{cm}^{3}\right)^{1 / 2}\right]$ & Ref \\
\hline Toluene & 2.4 & 8.8 & S5 \\
PDMS & 2.7 & 7.3 & S5 \\
Parylene E & $2.34^{18}$ & $8.4^{\mathrm{a}}$ & 18 , This work \\
\hline
\end{tabular}

${ }^{\mathrm{a}}$ estimated from Hansen solubility parameter ${ }^{\mathrm{S} 6}$ 


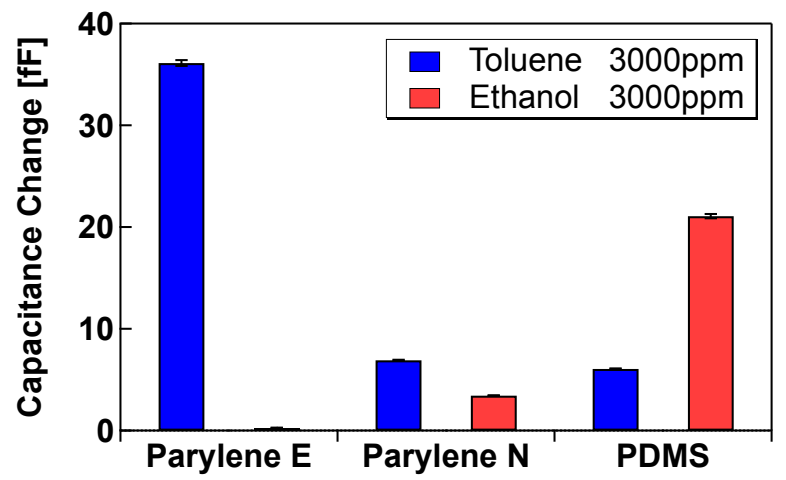

Figure S4. Equilibrium capacitance change of parylene E, parylene N and PDMS versus 3000 ppm of toluene (nonpolar VOC) and ethanol (polar VOC). A good selectivity between polar and non-polar VOC for parylene E is obtained. The selectivity factor, which is defined as the ratio between toluene and ethanol responses, is measured to be 130, 2.0, and 0.3 for parylene E, parylene N, and PDMS, respectively.

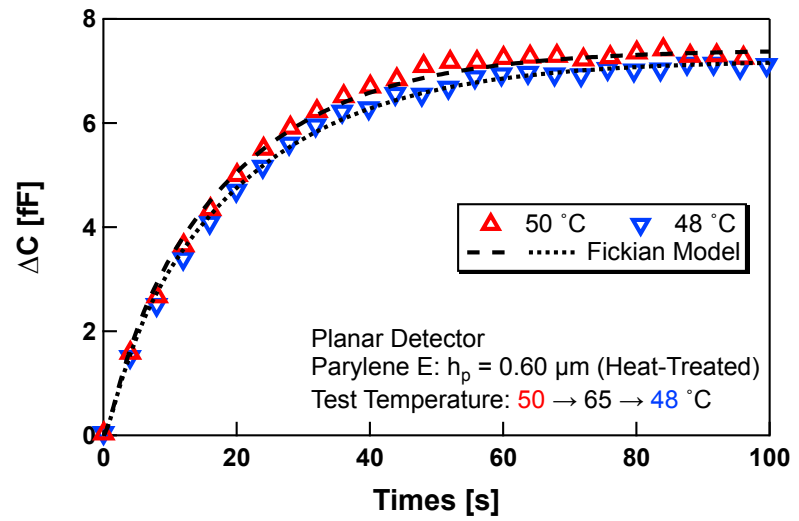

Figure S5. Investigation of hysteresis due to the temperature history. The temperature increases from room temperature to $50{ }^{\circ} \mathrm{C}$ for the first sorption test, and then increases to $65{ }^{\circ} \mathrm{C}$. The second sorption test is conducted after the temperature decreases from $65{ }^{\circ} \mathrm{C}$ to $48{ }^{\circ} \mathrm{C}$. The sorption profiles are fitted to the Fickian diffusion model. The differences in the diffusivity and the equilibrium capacitance change between two trials are $7 \%$ and $3 \%$, respectively. No obvious hysteresis of the sorption profiles between the heating and cooling cycles is observed. 


\section{S5. Simulation Details}

The gas diffusion in swollen polymers with moving boundary at the vapor-polymer interface is analyzed based on one-dimensional numerical model developed by Wu \& Peppas $(1993)^{[47,48]}$. The viscoelasticity of the polymer material is taken into account by the Maxwell model with the polymer relaxation time, $\tau_{\text {rel }}$. The diffusional Deborah number, $D e$, defined as the ratio of $\tau_{\text {rel }}$ to the diffusion time of VOC vapors, $\tau_{\text {diff }}$, is introduced for characterizing the diffusion process. The simultaneous differential equations are described in dimensionless form as below:

$$
\begin{gathered}
\frac{\partial \phi_{1}}{\partial \theta}=\left(1-\phi_{1}\right) \frac{\partial}{\partial \bar{X}}\left\{f\left(\phi_{1}\right) \frac{\partial \phi_{1}}{\partial \bar{X}}\right\}+\left(1-\phi_{1}\right) \frac{\partial}{\partial \bar{X}}\left\{g\left(\phi_{1}\right) \frac{\partial \bar{\sigma}}{\partial \bar{X}}\right\}, \\
f\left(\phi_{1}\right)=\left(1-\phi_{1}\right) \exp \left\{a_{\mathrm{d}}\left(\phi_{1}-\phi_{1, \mathrm{eq}}\right)\right\} \\
g\left(\phi_{1}\right)=\frac{\phi_{1} \exp \left\{a_{\mathrm{d}}\left(\phi_{1}-\phi_{1, \mathrm{eq}}\right)\right\}}{\left(1-2 \chi \phi_{1}\right)}, \\
\frac{\partial \bar{\sigma}}{\partial \theta}=-\frac{\bar{\sigma}}{D e \cdot \exp \left\{-a_{\eta} \phi_{1, \mathrm{eq}}\right\}}+\frac{\bar{E}}{\left(1-\phi_{1}\right)^{2}} \frac{\partial \phi_{1}}{\partial \theta} \\
D e=\frac{\tau_{\mathrm{rel}}}{\tau_{\mathrm{diff}}} \\
\tau_{\mathrm{rel}}=\frac{\eta}{E}=\frac{\eta_{0}}{E} \exp \left\{-a_{\eta} \phi_{1, \mathrm{eq}}\right\}
\end{gathered}
$$

where $\phi_{1}$ is the volume fraction, $\theta$ is the time normalized by $\tau_{\text {diff }}, \bar{X}$ is the length normalized by the polymer thickness, $\bar{\sigma}$ is the stress normalized by the normal stress in length direction, $a_{\mathrm{d}}$ is the coefficient in the exponent of the mutual diffusivity, $\phi_{1}$ is the equilibrium volume fraction, $\chi$ is the Flory-Huggins interaction parameter, $a_{\eta}$ is the coefficient in the exponent of the viscosity, $\bar{E}$ is the dimensionless Young's modulus, $E$ is Young's modulus, and $\eta_{0}$ is the viscosity before VOC sorption.

In the present analysis, a finite difference approach is adopted to solve Eqs. (S23) - (S28). A fully implicit time discretization scheme and the second-order central differencing for the space discretization are employed. A set of the nonlinear equations is linearized using a Newton method with multivariable Taylor expansion, and the discretized equation is solved based on the tridiagonal matrix algorithm (TDMA). Except the material properties, the Deborah number is only the free parameter. Based on the regression of the least root-mean-square (RMS) errors between the modeling result and the measured data, the Deborah number is obtained for each condition. 


\section{References (only for Supporting Information)}

[S1] D. Ballantine, Jr., R.M. White, S.J. Martin, A.J. Ricco, E.T. Zellers, G.C. Frye, H. Wohltjen, Acoustic Wave Sensors - Theory, Design, and Physico-Chemical Applications, Academic Press, 1996.

[S2] Stanford Research Systems, Operation and Service Manual: QCM200 Quartz Crystal Microbalance Digital Controller, 2.5 ed., 2018.

[S3] R. Igreja, C. J. Dias, Sens. Actuators, A: Phys. 2004, 112, 291.

[S4] R. Casalini, M. Kilitziraki, D. Wood, M.C. Petty, Sens. Actuators, B: Chem. 1999, 56, 37.

[S5] J.N. Lee, C. Park, G.M. Whitesides, Anal. Chem. 2003, 75, 6544.

[S6] C.M. Hansen, Hansen Solubility Parameters: A User's Handbook, 2nd ed., Taylor \& Francis, New York, USA 2007. 